\title{
Reorganización administrativa de una institución pública: Construcción de la estructura orgánica formal del Despacho del Gobernador del Estado de San Luis Potosí, México
}

\author{
ADMINISTRATIVE REORGANIZATION OF A PUBLIC INSTITUTION: CONSTRUCTION OF THE FORMAL \\ ORGANIZATIONAL STRUCTURE OF THE OFFICE OF THE GOVERNOR OF THE STATE OF SAN LUIS \\ POTOSÍ, MEXICO
}

\author{
Ismael Alavez Torres ${ }^{12}$ \\ Miguel Ángel Vega Campos ${ }^{13}$
}

\section{Resumen}

La elaboración de este trabajo surgió por la inquietud de documentar las acciones y estrategias que se ejecutaron para la reorganización administrativa del Despacho del Gobernador del Estado de San Luis Potosí, durante el periodo constitucional 1997-2003, a cargo de Fernando Silva Nieto. Se considera que un trabajo de estas características puede aportar evidencia empírica que sirva de referencia para otras organizaciones públicas -ya sean federales, estatales o municipales- que se interesen por la ejecución de acciones en el mismo sentido; es decir, puede ayudar desde el punto de vista jurídico, administrativo, técnico, metodológico, entre otros aspectos. Se trata de un trabajo realizado bajo un enfoque preponderantemente cualitativo, en virtud de que el mismo se apoyó casi en su totalidad de revisión documental, aunque también están inmersos en el estudio las experiencias y vivencias de los autores de esta investigación, quienes se desempeñaron como servidores públicos durante el periodo revisado y a quienes les tocó dirigir las acciones que aquí se documentan. Además de la descripción de las acciones y estrategias que se realizaron para el propósito de la reorganización, también se incluyen los productos que se generaron, derivados de dichas actividades.
En términos generales, la documentación del caso se realizó bajo la siguiente secuencia: primero se describe el entorno político administrativo de la dependencia y las actividades propias del titular del Poder Ejecutivo estatal, así como la problemática que presentaba el Despacho; segundo, se expone lo referente a la ejecución de un diagnóstico acerca de las condiciones prevalecientes en el ámbito interno de la unidad administrativa revisada; tercero, cómo es que se hizo frente a las deficiencias encontradas en cuanto la estructura orgánica, competencia y coordinación de funciones; y cuarto, se enuncia cuál fue la propuesta que se empleó para reestructurar la unidad administrativa objeto de estudio. EI trabajo realizado presenta diversas ventajas para varios actores, por ejemplo, los servidores públicos, los académicos e investigadores, los estudiosos de la ciencia política y la administración pública, entre otros; ya que la metodología aquí utilizada puede ser replicada para otras organizaciones públicas, independientemente del ámbito de gobierno de que se trate -federal, estatal o municipal-. Los análisis como el que aquí se trabajó, resultan escasos en el área de la administración pública y también en la profundidad con la que esta investigación se ejecutó. El hecho de documentar la experiencia vivida para realizar la reorganización del Despacho del Gobernador, ya resulta de por sí una aportación valiosa.

\footnotetext{
12 Licenciado en Ciencia Política y Administración Pública por la Universidad Nacional Autónoma de México (UNAM) y Maestro en Administración por la Universidad Autónoma de San Luis Potosí (UASLP). Se desempeña como Jefe de Departamento en el Gobierno del Estado de San Luis Potosí. Correo electrónico: ismaelalavez@yahoo.com.mx

${ }^{13}$ Maestro y doctor en administración por la Universidad Autónoma de San Luis Potosí. Se desempeña como Profesor investigador de tiempo completo en la Facultad de Contaduría y Administración de la UASLP, donde coordina la Maestría en Administración con Énfasis en Gestión Pública. Correo electrónico: vegacamposnet@hotmail.com
} 
Para los autores de este trabajo, resultó sumamente apasionante y enriquecedor, intervenir en la revisión de documentos oficiales generados durante el periodo gubernamental 1997-2003, cuestión que aportó todos los elementos necesarios para la realización de la investigación.

Palabras clave: Reorganización administrativa, organización pública, gobierno del Estado de San Luis Potosí, despacho del gobernador.

\section{Abstract}

The completion of this research arose from the concern of documenting the actions and strategies executed for the administrative reorganization of the Office of the Governor of the State of San Luis Potosí, during the constitutional period of 1997-2003, in charge of Fernando Silva Nieto. It is considered that a research with these characteristics can provide empirical evidence that serves as a reference for other public organizations -whether they are federal, state or municipal- with an interest in the execution of actions in the same sense, in other words, it can help from the legal, administrative, technological, technical, methodological point of view, among other aspects. It is a preponderantly qualitative research, taking into consideration the fact that is supported almost in its entirety by documentary review. However, the research also includes the experiences of the authors, who performed as public servants during the reviewed period and directed the actions documented in this research. In addition to the description of the actions and strategies utilized for the reorganization, the products generated derived from such activities are also included. In general terms, the documentation of the case was accomplished through the following sequence: first, the administrative political environment of the office and the activities of the state executive power holder are described, as well as the quandary the office represented; in second place, an exposition regarding the execution of a diagnosis of the conditions prevailing in the internal scope of the examined administrative unit; in third place, how the deficiencies found were dealt with in terms of organizational structure, competence and coordination of functions; and finally, the proposal used to restructure the administrative unit under study is stated.

The research shows diverse advantages for several actors, for instance, public servants, academics and researchers, scholars of political science and public administration, among others, since the methodology used can be replicated for other public organizations independently of the government sphere in question federal, state, municipal-. In the field of public administration, analysis such as the one made in this research, in the depth it was executed are very scarce. To document the experience of reorganizing the office of the Governor is in itself a valuable contribution. For the authors, it resulted extremely fascinating and rewarding, to intervene in the review of official documents generated during the governmental period of 1997-2003, fact that contributed to provide all the elements necessary to conduct this research.

Keywords: Administrative reorganization, public organization, state government of San Luis Potosí, Office of the Governor. 


\section{Introducción}

La sociedad es un cuerpo en constante movimiento y evolución, requiere por ello, que los mecanismos que guían su rumbo y desarrollo, se adecuen para dar respuesta en forma satisfactoria a su constante crecimiento y diversificación de necesidades; en consecuencia, la administración pública debe actualizar su estructura organizacional, de manera que ello le permita eficientar su desempeño para cumplir de manera efectiva la función y compromiso social que tiene encomendada.

Uno de los objetivos prioritarios de cualquier persona que esté al frente de una gubernatura, como lo es el caso del titular del Poder Ejecutivo del Estado de San Luis Potosí, es contar con un gobierno capaz de responder de manera efectiva y oportuna a las demandas que le plantea la sociedad. Esto hace necesario que las diversas dependencias y entidades que integran la administración pública de San Luis Potosí conozcan en forma precisa las atribuciones que les corresponden, así como la delimitación del marco jurídico que fundamenta su actuación, de tal manera que con ello se evite la duplicidad de funciones y el gasto innecesario de recursos -humanos, técnicos, financieros, materiales, tecnológicos, entre otros-.

\section{Planteamiento del problema}

Por lo anterior, resulta pertinente definir al Despacho del Gobernador del Estado de San Luis Potosí, como la unidad administrativa encargada de apoyar de manera técnica, humana, material, financiera y de información estratégica, para que dicho Despacho cumpla en condiciones óptimas, con el adecuado desarrollo de las funciones y actividades propias de la responsabilidad que tiene encomendada el titular del Ejecutivo del Estado, para atender y dar cumplimiento a los compromisos adquiridos con los potosinos desde el momento de su campaña política, y que le son inherentes a su investidura y responsabilidad, en la puntual observancia de sus atribuciones enmarcadas en la Constitución Política del Estado Libre y Soberano de San Luis Potosí (CPSLP) y en la Ley Orgánica de la Administración Pública del Estado de San Luis Potosí (LOAPSLP).
Ante tales circunstancias, el Despacho del Gobernador debe contar con el fundamento jurídico - administrativo que sustente la estructura orgánica de la institución y norme sus procesos de trabajo. En este sentido, el planteamiento del problema de investigación de este trabajo puede enunciarse en los siguientes términos: Revisar y documentar las acciones y estrategias que se desarrollaron durante el periodo 1997-2003, para la reorganización jurídico-administrativa del Despacho del Gobernador del Estado de San Luis Potosí.

\section{Objetivo general}

Generar evidencia empírica que permita conocer cuál fue el procedimiento que se ejecutó para la reorganización administrativa del Despacho del Gobernador del Estado de San Luis Potosí, implementada durante el periodo constitucional 19972003, de tal manera que la compilación documental que se integre sirva de marco metodológico de referencia para otros trabajos de características similares, que se ejecuten en cualquier otro tipo de organización pública.

\section{Marco teórico-conceptual}

Como parte del marco conceptual para sustentar este trabajo, resultó pertinente revisar determinados conceptos, los cuales se enuncian en los siguientes renglones.

Por principio de cuentas, encontramos que según el jurista mexicano González (2001), la conceptualización del Estado puede enunciarse como: 1) Una sociedad humana; 2) Establecida permanentemente en un territorio; 3) Regida por un orden supremo; 4) Bajo un orden jurídico; y 5) Que tiende a la realización de los valores individuales y sociales de la persona humana.

En el mismo tenor de ideas, González (2001) identifica los elementos del Estado, con el propósito de exponer una definición de manera integral. Dichos elementos deben considerarse dentro de una unidad, coexistiendo ordenadamente y no en contraposición unos con otros. 
En este caso, el primer elemento es la comunidad de hombres libres que en uso de su facultad soberana, es capaz de establecer una forma de organización política basada en las decisiones fundamentales que la propia comunidad ha tomado a lo largo de su evolución histórica.

En este punto, es conveniente distinguir otros elementos que tienen relación directa o indirecta con el término Estado. Dichos elementos son: nación, sociedad, población y pueblo. La nación es el conjunto de individuos con vínculos como lengua, religión, raza, entre otros; con conciencia de pertenecer a la misma comunidad.

Por lo que hace al concepto de sociedad se refiere a los fines comunes que pretende alcanzar la agrupación humana localizada en un territorio específico. Población es un concepto aritmético para la identificación numérica de los habitantes. La noción pueblo se refiere específicamente a la comunidad de hombres vinculados políticamente con el Estado. El segundo elemento es la permanencia y estabilidad de ese conglomerado humano radicado en un territorio cuyos límites se encuentran claramente determinados.

El tercer elemento es el orden jurídico, que es la expresión formal de toda la organización política y el sistema encargado de regular las relaciones de todos los miembros de la comunidad (gobernados y gobernantes). Es, asimismo, el elemento que garantiza la permanencia de la comunidad y le permite resolver conflictos en forma pacífica y ordenada con base en procedimientos institucionales.

El cuarto elemento es el gobierno, compuesto por diversos órganos cuyos titulares están encargados de garantizar la vigencia del orden jurídico, para lo cual cuentan con el poder de mando y coerción necesarios (sin coacción no hay derecho). No debe confundirse este poder gubernamental con el poder de la unidad total llamado Estado.
El quinto elemento es el poder del Estado, que surge de la conjugación, interacción y manifestación dinámica de los elementos anteriores, y que es la potestad o fuerza máxima de la cual derivan su poder todas las demás organizaciones inferiores de la sociedad. Dicho poder garantiza la existencia del Estado frente a las demás comunidades estatales (internacionales). Este poder es indivisible, en tanto que el poder gubernamental sí se divide, para su ejercicio, entre los diversos órganos gubernamentales.

Se expone un sexto elemento que es la legitimidad, describiendo que la organización estatal sea el producto de una decisión de voluntad popular, de una decisión de la comunidad que establece la organización política para garantizar su convivencia pacífica y la realización de los valores individuales y sociales de la persona humana (el bien de la comunidad).

En este entendido se puede citar un séptimo elemento, que Aristóteles al referirse a la finalidad de la polis, señalaba que ésta es la comunidad política a la que llamamos ciudad. González (2001) afirma que el Estado tiende a la consecución de fines concretos y particulares que, al fin de cuentas, se unifican en un fin total omnicomprensivo: el bien de la comunidad entera.

La síntesis que surge de los elementos del Estado es la siguiente: una comunidad de hombre libres unidos por vínculos de solidaridad nacional, localizada en un territorio determinado, organizada políticamente bajo un gobierno con unidad de mando y sujeta a un orden jurídico nacional, que tiende a la realización de los fines y metas sociales de la comunidad entera. La unidad Estado es distinta de sus elementos aun cuando surja de ellos. Sus fines son propios y diversos de los de cada uno de sus miembros. 
El Estado sobrevive a las variaciones de sus elementos. El Estado es un sujeto de derechos y por lo tanto una persona jurídica. Es el medio más idóneo que el hombre ha encontrado para la consecución de los fines sociales.

Pasando al término de Gobierno, Orlandi (1996) afirma que la idea de gobierno es una de las que posee mayor antigüedad y centralidad dentro de la historia del pensamiento político, la razón es que esta palabra se encuentra estrechamente relacionada con la definición de política. Por su parte, Maurizio Cotta, (citado en Zaviris, A, 2012 p. 31) señala que "pueden faltar los partidos, los parlamentos, las elecciones, los aparatos burocráticos desarrollados, pero no una estructura de gobierno". Por lo que se refiere a la descripción etimológica del término gobierno, encontramos que la palabra griega Kibernao - de la que deriva gobiernosignifica dirigir el timón, aludiendo a la docilidad de la nave al timón (gobernalle o gobernallo). Esta raíz es la que mejor nos acerca al concepto de gobierno. De esta manera podemos deducir que el timonel que conduce con certeza la nave, la llevará a buen puerto, entonces estamos hablando de la ejecución de un buen gobierno.

En la edad media el término gubernaculum era identificado con el de política Sartori, 1987, (citado en Zaviris, A, 2012 p. 31). La llegada de la democracia impulsó en fechas posteriores la evolución del concepto gobierno, al mismo tiempoque ocasionó la multiplicidad de imágenes, incorporando al pueblo (soberano) como eje de las mismas (la idea de voluntad general), y a los gobernantes, como meros representantes. El gobierno en verdad tiene una "función identificadora de la actividad política, [es] el núcleo irreductible, sin el cual parece difícil pensar en la dimensión propia de lo político" (Orlandi, 1996).

Según Rojas 1971, (citado en Zaviris, A, 2012) el gobierno dirige la vida de una nación, por medio de mandatos, recomendaciones y con la cooperación de los gobernados. El gobierno manda por medio de leyes y demás disposiciones de carácter general; pero también puede tomar decisiones de carácter particular como decretos, acuerdos, órdenes, instrucciones y decisiones administrativas.
El gobierno tiene a su cargo la tarea de crear el orden jurídico.

La fuerza material de que dispone el Estado es un medio para asegurar la ejecución o el cumplimiento de sus determinaciones. La principal fuerza de que dispone el Estado está en la adhesión de los gobemados que, integrando a la opinión pública, orientan la acción pública de los gobernantes. La fuerza material es necesaria, pero es una medida transitoria y de excepción. Un gobierno apoyado en la fuerza es siempre un gobierno inestable y tiránico. En términos generales se acepta que "el gobierno es la organización que representa al Estado para el cumplimiento de sus objetivos y metas" (Castelazo, 2007).

Comprender el concepto de gobierno implica conjugar factores conceptuales tendientes a argumentar cuestiones tan importantes como su integración, funcionamiento, su papel en la resolución de conflictos y en la toma de decisiones, y la evaluación social y política de su desempeño, entre otros aspectos.

Castelazo (2007, p. 25) afirma que una manera más para explicar al gobierno es por su función y otra es por su expresión orgánica. Expone el citado autor que la función del gobierno es complejísima, ya que a grandes rasgos significa la organización del dominio en una sociedad. La efectividad y profundidad del gobierno dependen en gran medida de cómo se encuentra estructurado. Dicho de otra forma, función y organización son dos caras de la misma moneda. El autor cuestiona: ¿a quién sirve el gobierno?, entonces agrega: en los grupos primitivos el dominio fue el más fuerte. A través del tiempo, dicho dominio fue combinándose, primero con la tradición y finalmente como históricamente lo demuestra Weber- con la ley. Es evidente que fuerza, carisma, tradición y legalidad se combinan para conformar una entidad que tiene el poder, en este caso el gobierno, misma que es la encargada de hacer y actualizar leyes (Poder Legislativo), ejecutar sus ordenamientos (Poder Ejecutivo), e interpretar las normas para la aplicación de la justicia (Poder Judicial). 
En virtud de estas condiciones, Castelazo (2007) continúa explicando la autoridad del gobierno, posee legitimidad, autonomía y soberanía no por sí misma, sino en representación del conjunto social. Por lo tanto, el gobierno sirve a todos, pues el mundo actual es complejo y se refleja en todos los ámbitos, no puede basarse en un solo interés, sino atendiendo al equilibrio socioeconómico y político, procurando que la generación y producción de riqueza y bienestar sea equitativa en su distribución y beneficios.

Otro concepto que vale la pena revisar es el de "administración pública". Por lo que a éste se refiere, la Real Academia Española de la Lengua señala lo define como "la acción del gobierno de dictar y aplicar las disposiciones necesarias para el cumplimiento de las leyes y para la conservación y fomento de los intereses públicos $y$, al resolver las reclamaciones a que dé lugar lo mandado. Es el conjunto de organismos encargados de cumplir esta función".

Por otro lado, Moreno (1979) refiere que la administración pública, en tanto estructura orgánica, es una creación del Estado, regulada por el derecho positivo, y como actividad constituye una función pública establecida por el ordenamiento jurídico nacional. Pero tanto la organización como la función o actividad reúnen, además, caracteres tecnicopolíticos, correspondientes a otros campos de estudios no jurídicos, como los de la teoría de la organización administrativa y la ciencia política.

Por lo tanto, la noción de administración pública dependerá de la disciplina o enfoques principales de estudio (el jurídico, el técnico o el político), en virtud de no existir una ciencia general de la administración pública, capaz de armonizar y fundir todos los elementos y enfoques de estudio de este complejo objeto del conocimiento. Hasta el momento no es posible dar una noción omnicomprensiva de la administración pública, porque ello equivaldría a mezclar fenómenos diferentes, autónomos e independientes.
La teoría de la organización concibe a la administración pública como una organización; una organización humana que tiende a la consecución de múltiples propósitos políticos, económicos y sociales, a través del aprovechamiento eficiente y productivo de los recursos disponibles y mediante la utilización e interacción de los diversos sistemas, procedimientos, mecanismos y recursos con que cuenta. Dicha organización tiene que ver con cuestiones sumamente importantes, como las siguientes: el proceso de toma de decisiones; la planeación y programación de actividades; la presupuestación; la administración eficiente del personal y de los recursos materiales y financieros; las técnicas de organización y métodos administrativos; la informática; la contabilidad; la evaluación y el control.

\section{Metodología de la investigación}

\section{Enfoque de la investigación}

Esta investigación se realizó bajo un enfoque eminentemente cualitativo, el cual utiliza la recolección de datos sin medición numérica para descubrir o afinar preguntas de investigación en el proceso de interpretación. Es importante mencionar que en los estudios cualitativos no existe un patrón a seguir para desarrollar las preguntas de investigación, ya que se pueden desarrollar antes, durante o después de la recolección y el análisis de datos. En términos de Hernández, Fernández y Baptista (2010), "estas actividades sirven, primero, para descubrir cuáles son las preguntas de investigación más importantes, y después, para refinarlas y responderlas.

Tipo de investigación

Este trabajo se trató de un tipo de investigación documental, ya que como bien lo señala Bernal (2010, p. 111), "la investigación documental consiste en un análisis de la información escrita sobre un determinado tema, con el propósito de establecer relaciones, diferencias, etapas, posturas o estado actual del conocimiento respecto al tema objeto de estudio". 
Como parte del trabajo que aquí se presenta, se recopiló información contenida en archivos, documentos normativos como leyes, reglamentos, decretos, acuerdos administrativos, entre otros. La investigación se supeditó totalmente a datos escritos. En este sentido, de acuerdo con Casares Hernández et al. (1995; citados por Bernal, 2010, p. 111), "la investigación documental depende fundamentalmente de la información que se obtiene o se consulta en documentos, entendiendo por éstos todo material al que se puede acudir como fuente de referencia, sin que se altere su naturaleza o sentido, los cuales aportan información o dan testimonio de una realidad o un acontecimiento", como en este caso fue la reorganización administrativa que implicó el Despacho del Gobernador del Estado de San Luis Potosí, la cual se realizó durante el periodo 1997-2003.

\section{Análisis y discusión de resultados}

Para el análisis que aquí se presente, se revisaron los datos encontrados sobre el Despacho del Gobernador, para determinar primero, cuáles fueron las fallas administrativas más ostensibles que obstaculizaban su desenvolvimiento de operación para posteriormente, señalar cuáles fueron las medidas implementadas para hacer frente a las deficiencias localizadas en su estructura y funcionamiento.

Se observa que la labor de diagnosis se circunscribió al campo de los organismos y unidades administrativas adscritas al Despacho del titular del Ejecutivo Estatal, al inicio de la administración de Fernando Silva Nieto como Gobernador Constitucional del Estado. En este caso, vale la pena señalar que la esfera de investigación concerniente a los aspectos administrativos de las estructuras del gobierno del Estado, es tema apenas explorado, y representa, por su trascendencia y complejidad, un reto para los futuros intentos de mejorar operativamente la administración pública de San Luis Potosí.

\section{Deficiencias encontradas en la coordinación} administrativa del Ejecutivo
El crecimiento del Gobierno del Estado de San Luis Potosí, ha dado origen a una alta multiplicidad de instituciones y organismos que atienden a programas específicos con influencia directa en el desarrollo económico de San Luis Potosí. Evitar las duplicaciones, las lagunas de responsabilidad e integrar armónicamente la acción del Estado, constituye una tarea necesaria y urgente. La coordinación de dichas unidades administrativas, responsables de la actividad pública, comprende tanto la conexión al nivel de las dependencias del Ejecutivo, como la integración de las funciones dentro de cada Secretaría de Estado; implica, asimismo, la coordinación de los programas de los organismos desconcentrados y descentralizados con los órganos centrales del Poder Ejecutivo.

Resulta axiomático que en la medida en que se desarrolla el esfuerzo especializado por la complejidad creciente de las actividades del Gobierno del Estado, mayor es el imperativo de coordinar la acción para imprimir al trabajo conjunto del Gobierno, un sentido de unidad y de propósito. Está plenamente comprobado por la experiencia- que la clave de la eficiencia operativa radica en la coordinación de los recursos y esfuerzos; y en la esfera gubernamental, la armonización de las políticas del Estado, es la clave de la programación económica y social.

Por ser tan obvia la necesidad de coordinar la acción en todas las manifestaciones de la vida institucional, la integración del esfuerzo en la administración pública encuentra grandes obstáculos, debido, a veces, a la persistencia del factor político, pero en la mayoría de los casos, por la forma desorganizada de las unidades administrativas y los servicios públicos. A juicio de los autores, se mencionan las principales fallas de coordinación en la rama ejecutiva del Gobierno del Estado de San Luis Potosí, las cuales se describen en lo sucesivo. 
a) Métodos deficientes de comunicación interinstitucional

La Secretaría Técnica del Gabinete del Despacho del Gobernador, según expresión más conocida, no actúa como organismo de coordinación, ya que no reúne a instituciones afines para dar solución a los problemas que enfrenta día a día el Ejecutivo del Estado; quedando sólo como recurso de coordinación superior del Gobierno, a los "acuerdos administrativos" que individualmente celebran los titulares de las dependencias y algunas reuniones esporádicas de los titulares de las secretarías con funciones económicas. El Informe de Gobierno en algo contribuye a la tarea de armonizar el trabajo de la administración pública centralizada y la paraestatal, pero evidentemente en forma esporádica y limitada.

b) Formas débiles de colaboración en el régimen interno de las dependencias del Ejecutivo

El factor político influye en la coordinación de las unidades administrativas de cada dependencia. Cierto es que la autoridad es el centro coordinador por excelencia en toda organización, pero cuando las líneas de mando no están bien definidas, los resultados no alcanzan la efectividad deseada. No existen generalmente las bases de colaboración recíproca, ni la información interdepartamental, ni la disciplina del trabajo en equipo que permitan y auspicien el desarrollo de una acción armónica y más eficiente.

c) Deficiente integración de los organismos descentralizados con los órganos centrales del poder público

Las coordinaciones generales que se han insertado al Despacho del titular del Ejecutivo, son productos de "amarres" políticos por parte de sus propios titulares, y nunca pensando en la cohesión, integración y control de este tipo de entidades del gobierno, en sus relaciones con las entidades centrales de la administración pública estatal. d) Falta de coordinación en los procesos operativos

La administración gubernamental se manifiesta en una serie de procesos que cubren una amplia esfera de funciones sustantivas y auxiliares de responsabilidad estatal. Se ha destacado en el curso de este trabajo acerca de la importancia de coordinar las políticas económicas y sociales, como base para iniciar la acción planificada del Gobierno. Como complemento de ese esfuerzo, debe señalarse la conveniencia de coordinar los procesos administrativos en los principales aspectos de la actividad gubernamental. Es decir, coordinar las normas metodológicas de conducir las funciones de las entidades del Gobierno del Estado en materia presupuestal, compras, contratación del personal, inventarios, etcétera.

\section{e) Deformaciones del régimen funcional}

La base de la organización de la administración pública de San Luis Potosí se sustenta en el artículo $3^{\circ}$ de la Ley Orgánica de la Administración Pública del Estado, fuente originaria de atribuciones para cada uno de los grandes compartimientos de que se compone. El contenido de responsabilidad, aparece en este ordenamiento en forma macro-administrativa y para el cumplimiento de estas atribuciones, se provee el órgano de ejecución que ha de realizarlas. El examen del régimen administrativo desde el ángulo funcional, nos lleva a consignar las deformaciones que se enuncian en los siguientes puntos.

f) Excesiva centralización de funciones en los niveles superiores de la jerarquía administrativa del gobierno

El Gobernador del Estado, el titular del Despacho, los coordinadores generales y otros funcionarios superiores, se ven abrumados de asuntos de detalle, firmas autógrafas y audiencias, por lo cual dejan poco tiempo para la formulación de las decisiones de política directiva. El recurso de delegar funciones se ve trabado por formalismos legales que demandan atención directa y personal de los ejecutivos principales, en una multiplicidad de asuntos oficiales y la práctica más ostensible, es la dirección altamente centralizada de la actividad gubernamental. 


\section{g) Duplicidad de funciones}

Para citar sólo un ejemplo, señalaremos que en materia de captación de solicitudes y demandas de la ciudadanía, así como su respuesta correspondiente, son las siguientes unidades administrativas adscritas al Despacho, las realizan esta actividad: 1) Dirección General Ejecutiva; 2) La Dirección de Gestión y Atención Ciudadana; 3) La Secretaría Técnica del Gabinete; 4) La Dirección General de Comunicación Social; 5) La Coordinación General de Proyectos Estratégicos; y 6) El Sistema de Financiamiento para el Desarrollo del Estado (SIFIDE).

h) Imprecisión de las esferas de competencia

Aunque la Ley Orgánica de la Administración Pública del Estado de San Luis Potosí delimita las atribuciones de cada dependencia del Ejecutivo Estatal, subsisten de hecho ambigüedades en varios campos de la administración pública que provocan fricciones e invasión de funciones. Aun cuando el Artículo 14 de la citada Ley, expresa que: "Cuando existan dudas respecto a la competencia de las dependencias y entidades para la atención de algún asunto, el Gobernador del Estado decidirá a cuál de ellas corresponde y emitirá, en su caso, el acuerdo respectivo que limite la esfera de su competencia".

i) Indiferenciación de las funciones de línea y las funciones de "staff"

Esta distinción en que se basa una de las técnicas más importantes de la ciencia de la administración al separar las actividades de apoyo, respecto a las de ejecución, no se ha aprovechado adecuadamente en el campo de la función pública potosina. La complejidad de las instituciones ha determinado que, de acuerdo con el principio de división del trabajo, se incorporen organismos asesores para auxiliar el trabajo de los ejecutivos, cuya función no se manifiesta en forma de instrucciones o de órdenes, sino en aportación de ideas e información para apuntalar las decisiones y dar vitalidad a la organización interna de las instituciones. j) Heterogeneidad de las funciones asignadas a algunas dependencias del Ejecutivo estatal

Aunque mucho se ha avanzado al delimitar los campos de acción de las dependencias gubernamentales, de acuerdo con un criterio de homogeneidad, subsisten aún actividades dentro de algunas dependencias $u$ organismos que no corresponden a la naturaleza propia de sus funciones, deformando consecuentemente, el régimen funcional del Gobierno del Estado. Un caso muy claro es la Coordinación General de Proyectos Estratégicos, la Coordinación para asuntos especiales de la Zona Huasteca y la Coordinación General del Transporte Urbano para la Zona Metropolitana de San Luis Potosí, cuyas funciones esenciales corresponden a las Secretarías de Planeación del Desarrollo, a la Secretaría General de Gobierno y a la Secretaría de Desarrollo Urbano, Comunicaciones y Transportes respectivamente.

k) Fallas en la estructura orgánica

La evolución de la estructura administrativa del Gobierno de San Luis Potosí, nos permite apreciar cómo el desarrollo institucional de Poder Ejecutivo está ligado a los acontecimientos históricos más importantes de nuestro país. En las últimas décadas, y con una sola excepción, al iniciarse cada régimen de gobierno, se introducen cambios en la estructura del Poder Ejecutivo con la mira de adaptar una política de trabajo acorde a los planes nacionales de desarrollo.

En presencia de este panorama estructural del Poder Ejecutivo del Estado y de las situaciones que se han creado por la magnitud y complejidad de la administración pública para la realización de los programas y planes estatales, podríamos apuntar las siguientes fallas de la estructura gubernamental: a) Crecimiento sin obediencia a un plan, b) Excesiva cantidad de acuerdos directos del Gobernador, c) Falta de uniformidad en la estructura interna de las dependencias del Ejecutivo del Estado. Generalmente la Administración Pública Centralizada, d) Ausencia de oficinas de planeación, y de organización y métodos a nivel jerárquico adecuado; y e) Pulverización de la estructura gubernamental. 


\section{Conclusiones y recomendaciones}

Una cuestión que resulta pertinente resaltar de este trabajo, es que se tuvo la ventaja de interpretar las vivencias de los servidores públicos que han prestado sus servicios en el gobierno del Estado, desde el periodo gubernamental del Profesor Carlos Jonguitud Barrios. Para los autores de este trabajo, resultó sumamente apasionante y enriquecedor, intervenir en la revisión de documentos oficiales durante el periodo de gubernamental comprendido entre los años de 1997 al año 2003.

De contar con la anuencia para realizar cambios de titulares en las direcciones que revisten alguna inconsistencia o problemática, se lograría un efecto de demostración muy positivo, e inclusive, también para el personal técnico, operativo y de apoyo. En su defecto, de no tener esta posibilidad, se deberán realizar acciones programadas o no, para medir la eficiencia de los planes, metas y acciones de las mismas y responsabilizar a los titulares, a fin de que nomen sus acciones conforme a un verdadero sistema de evaluación del desempeño de la propia institución.

Cabe resaltar la actitud sistemática de algunos titulares de esta dependencia, que por encima de cualquier instrumento jurídico, emplean los "argumentos" de: "por instrucciones superiores", "lo pide el Señor Gobernador", "si no autorizas, le digo al Gobernador" y "el peticionario es cuate" del Ejecutivo Estatal. Estas actitudes dañan la imagen institucional de la Secretaría y la del propio Titular del Ejecutivo, y lo más grave, los contratiempos que generan para "justificar" presupuestalmente estas prácticas administrativas, por lo que se recomienda su erradicación.

El Gobierno del Estado no realiza inversiones ni obra pública como en anteriores regímenes, debido en parte a que el Gobierno Federal les otorga a los ayuntamientos la operación y manejo de los recursos provenientes del Ramo 33, por lo que es necesario que el mandatario potosino siga manteniendo comunicación y contacto directo con la gente y sus necesidades, de esta manera fortalece su imagen y la de las instituciones de la administración pública estatal, obtiene reconocimiento de la ciudadanía y proyecta credibilidad en sus acciones de gobierno; se sugiere que este contacto se enfoque con las necesidades que demandan las nuevas condiciones políticas y sociales del estado.

Es importante señalar que uno de los proyectos impostergables, es contar con una red interna que permita generar procesos efectivos de trabajo, para contar con información veraz y oportuna. Con este fin se deberán diseñar paquetes para administrar los recursos humanos y el presupuesto de egresos, así como de peticiones que la ciudadanía envía al Ejecutivo, entre los más importantes.

\section{Referencias bibliográficas}

Bernal, C. A. (2010). Metodología de la investigación: administración, economía, humanidades y ciencias sociales. Colombia: Pearson, tercera edición.

Castelazo, J. R. (2007). Administración pública: una visión de Estado. México, Distrito Federal: Instituto Nacional de Administración Pública, A. C.

Gobierno del Estado de San Luis Potosí. (1979). Ley Orgánica de la Administración Pública del Estado de San Luis Potosí. San Luis Potosí: Periódico Oficial del Estado, 11 de octubre de 1979, Decreto No. 179, suplemento al No. 81.

Gobierno del Estado de San Luis Potosí. (1993). Ley Orgánica de la Administración Pública del Estado de San Luis Potosí. San Luis Potosí: Periódico Oficial del Estado, 29 de marzo de 1993, Decreto No. 722.

Gobierno del Estado de San Luis Potosí. (1997). Ley Orgánica de la Administración Pública del Estado de San Luis Potosí. San Luis Potosí: Periódico Oficial del Estado, 24 de octubre de 1997, Decreto No. 7.

González, H. (2001). Teoría política. México: Editorial Porrúa. 
Hernández, R., Fernández, C \& Baptista, P. (2010). Metodología de la investigación. México: Mc Graw Hill, quinta edición.

Moreno, R. (1979). La administración pública federal en México. México: Universidad Nacional Autónoma de México, Instituto de Investigaciones Jurídicas, Serie G, Estudios Doctrinales 45.

Orlandi, H. (1996). Introducción a la ciencia política. Buenos Aires, Argentina: Editorial Universitaria de Buenos Aires.

Zaviris, A. (2012). La comunicación gubernamental en los Ayuntamientos de Veracruz, periodo 2008-2010. Veracruz, México: Tesis Doctoral, Eumed Net. 